CI: 1.9-2.3) compared with metropolitan areas; increased by 6\%(95\% CI: $5 \%-7 \%)$ per decile of increasing disadvantage of postcode; and were higher in women aged 15-24 (AOR = 2.4,95\% CI: 2.3-2.6) and 25-34 years $(\mathrm{AOR}=1.7 ; 95 \% \mathrm{CI}$ : 1.6-1.8) compared with 35-45 years. Ectopic pregnancy rates in females were higher in inner-regional $(\mathrm{AOR}=1.3 ; 95 \%$ CI: $1.2-1.4)$ and outer regional $/$ remote $(\mathrm{AOR}=1.7 ; 95 \% \mathrm{CI}$ : $1.5-1.8$ ) areas; increased by 6\% (95\% CI: 5\%-7\%) per decile of increasing disadvantage; and were highest among 25-34 year-old women (AOR: 2.1; 95\% CI: 2.0-2.3). In men, epididymitis rates were higher in inner-regional $(\mathrm{AOR}=1.6 ; 95 \%$ CI: $1.5-1.7)$ and outer regional $/ \mathrm{remote}(\mathrm{AOR}=2.3 ; 95 \% \mathrm{CI}$ : 2.2-2.5) areas and did not differ by age or SES.

Conclusion Possible explanations for higher ED rates of chlamydia sequelae in non-metropolitan Australian residents could be higher chlamydia prevalence or variable access to primary healthcare in these areas.

Disclosure of interest statement These data are being analysed as part of the Australian Chlamydia Control Effectiveness Pilot (ACCEPt) study funded by the Commonwealth Department of Health and the National Health and Medical Research Council.

\section{P08.19 RISK OF PELVIC INFLAMMATORY DISEASE FROM CHLAMYDIA AND GONORRHOEA AMONG AUSTRALIAN SEXUAL HEALTH CLINIC ATTENDEES}

${ }^{1} \mathrm{JL}$ Goller*, ${ }^{2} \mathrm{CK}$ Fairley, ${ }^{2} \mathrm{CS}$ Bradshaw, ${ }^{1} \mathrm{AM}$ De Livera, ${ }^{2} \mathrm{MY}$ Chen, ${ }^{3} \mathrm{RJ}$ Guy, ${ }^{1} \mathrm{JA}$ Simpson, ${ }^{1}$ JS Hocking. 'Melbourne School of Population and Global Health, University of Melbourne; ${ }^{2}$ Central Clinical School, Monash University and Melbourne Sexual Health Centre; ${ }^{3}$ Kirby Institute, UNSW, Australia

\subsection{6/sextrans-2015-052270.365}

Background Pelvic inflammatory disease (PID) is an important cause of infertility in women and can occur when micro-organisms such as chlamydia or gonorrhoea ascend to the upper genital tract. However few studies have quantified the contribution of these pathogens to PID.

We estimated the burden of PID using the population attributable risk percent (PAR\%) in an Australian urban sexual health clinic population that could potentially be avoided if chlamydia or gonorrhoea infection were prevented.

Methods Data were extracted from the clinic's electronic patient database for all females aged 16-49 at first visit to an urban sexual health clinic between Jan2006-Jun2013. Chlamydia and gonorrhoea tests were based on clinical and risk assessment. PID diagnosis was based on clinical examination findings. Two analyses were undertaken; one among chlamydia-tested women and one among a subset of the chlamydia-tested group who were also tested for gonorrhoea (chlamydia/gonorrhoea tested). Univariable and multivariable logistic regression was conducted to identify factors associated with PID. The PAR\% for PID from a current chlamydia or gonorrhoea infection was calculated and adjusted for demographic and behavioural factors using multivariable logistic regression.

Results Among 15690 chlamydia-tested women, 1279 (8.2\%, 95\% CI 7.7-8.6) tested chlamydia-positive, $436(2.8 \%, 95 \%$ CI 2.5-3.0) had PID diagnosed. The PAR\% for chlamydia was 14.1\% (95\% CI 9.9-18.1). Among 8839 chlamydia/gonorrhoeatested women, $681(7.7 \%, 95 \%$ CI 7.2-8.3) had chlamydia only, $30(0.3 \%$, 95\% CI $0.2-0.5)$ gonorrhoea only, 22(0.2\%, $95 \%$
CI 0.2-0.4) chlamydia and gonorrhoea; 419 (4.7\%, 95\% CI 4.3-5.2) had PID diagnosed. The PAR\% was highest for chlamydia only $(12.5 \%$, 95\% CI 8.5-16.3) compared with gonorrhoea only $(0.9 \%, 95 \%$ CI-0.1-1.8) or concurrent infections $(1.0 \%$, 95\% CI 0.0-1.9).

Conclusion In this low gonorrhoea prevalence population, chlamydia control would have the greatest impact on reducing PID.

Disclosure of interest statement The authors declare that they have no commercial or other association that might pose a conflict of interest.

\section{P08.20 PATHOGEN NEGATIVE PELVIC INFLAMMATORY DISEASE: IS IT PID?}

${ }^{1} \mathrm{JL}$ Goller*, ${ }^{2} \mathrm{CK}$ Fairley, ${ }^{2} \mathrm{CS}$ Bradshaw, ${ }^{1} \mathrm{AM}$ De Livera, ${ }^{2} \mathrm{MY}$ Chen, ${ }^{3} \mathrm{RJ}$ Guy, ${ }^{1} \mathrm{JS}$ Hocking. ${ }^{1}$ Melbourne School of Population and Global Health, University of Melbourne; ${ }^{2}$ Central Clinical School, Monash University and Melbourne Sexual Health Centre; ${ }^{3}$ Kirby Institute, UNSW, Australia

\subsection{6/sextrans-2015-052270.366}

Background Pelvic inflammatory disease (PID) occurs when pathogens (often sexually transmitted) ascend from the cervix to the upper genital tract. No pathogen is detected in up to two thirds of PID cases yet few studies have assessed the characteristics of pathogen-negative PID. We assessed the characteristics of pathogen-negative compared to pathogen-positive PID using data from females attending a large urban sexual health clinic in Melbourne.

Methods Data were extracted from the clinic's electronic patient database for women aged 16-49 and tested for chlamydia (CT), gonorrhoea (NG), mycoplasma genitalium (MG) and bacterial vaginosis (BV) and diagnosed with PID at first clinic visit between Jan 2006-June 2013. PID diagnosis was clinical: based on uterine, cervical motion, or adnexal tenderness in sexually active women with pelvic pain where no cause beside PID was identified. Multivariable logistic regression was conducted to identify demographic, sexual behavioural factors and whether vaginal inflammation (defined as $\geq 5$ polymorphonuclear leukocytes (PMNL) per 1000 high powered field from high vaginal swabs) was associated with pathogen- negative PID.

Results Between 2006-2013, a total of 326 new females patients were diagnosed with PID and tested for CT, NG, MG and BV; $203(62 \%$; 95\% CI: 57\%-68\%) tested negative for the four infections (pathogen-negative PID). Among pathogen-positive PID cases, 49.6\% (95\% CI: 40.6-58.6) had CT, 6.5\% (95\% CI: 2.1-10.9) had NG, 12.2\% (95\% CI: 6.3-18.1) had MG, 56.1\% (95\% CI: 47.2-65.0) had BV. Multivariate analysis showed that pathogen-negative PID cases were less likely to have vaginal inflammation ( $\mathrm{OR}=0.5,95 \% \mathrm{CI}: 0.3-0.8)$, to report unprotected sex with non-regular sexual partners in the past three months $(\mathrm{OR}=0.6,95 \% \mathrm{CI}: 0.4-1.0)$ or to present as an STI contact $(\mathrm{OR}=0.4,95 \% \mathrm{CI}: 0.2-1.0)$.

Conclusion These findings suggest that pathogen-negative PID is associated with a lower sexual risk and inflammation then pathogen-positive PID cases. In the clinical setting, consideration of PMNS in diagnosing PID may improve diagnostic precision.

Disclosure of interest statement The authors declare that they have no commercial or other association that might pose a conflict of interest. 